\title{
A generalization of deferred Cesáro means and some of their applications
}

Uğur Deǧer* and Mehmet Küçükaslan

${ }^{\text {*Correspondence: }}$ degpar@hotmail.com

Department of Mathematics, Faculty of Science and Literature, Mersin University, Mersin, 33343, Turkey

\begin{abstract}
The deferred Cesáro transformation, which has useful properties not possessed by the Cesáro transformation, was considered by RP Agnew in 1932. The aim of this paper is to give a generalization of deferred Cesáro transformations by taking account of some well-known transformations and to handle some of their properties as well. On the other hand, we shall consider the approximation by the generalized deferred Cesáro means in a generalized Hölder metric and present some applications of the approach concerning some sequence classes.
\end{abstract}

MSC: Primary 40Gxx; 41A25; secondary 42A10

Keywords: deferred Cesáro method; Hölder metric; trigonometric polynomials; almost monotone sequences; degree of approximation

\section{Definitions and some notations}

Assume that $f$ is a $2 \pi$-periodic function and $f \in L_{p}:=L_{p}[0,2 \pi]$ for $p \geq 1$ where $L_{p}$ consists of all measurable functions for which the following, denoting the $L_{p}$-norm with respect to $x$, is finite:

$$
\|f\|_{p}:=\left\{\frac{1}{2 \pi} \int_{0}^{2 \pi}|f(x)|^{p} d x\right\}^{\frac{1}{p}}<\infty .
$$

Let

$$
f \sim \frac{a_{o}}{2}+\sum_{k=1}^{\infty}\left(a_{k} \cos k x+b_{k} \sin k x\right) \equiv \sum_{k=0}^{\infty} A_{k}(f ; x)
$$

be the Fourier series of a function $f \in L^{1}$. The partial sum of the first $(n+1)$ terms of the Fourier series of $f \in L_{p}$ at a point $x$ is denoted by

$$
S_{n}(f ; x)=\frac{1}{2} a_{0}+\sum_{k=1}^{n}\left(a_{k} \cos k x+b_{k} \sin k x\right) \equiv \sum_{k=0}^{n} A_{k}(f ; x) .
$$

Furthermore, a function $f$ is said to belong to the $\operatorname{Lip}(\alpha, p)$ class for $0<\alpha \leq 1$ and $p \geq 1$ if $\omega_{p}(\delta, f)=O\left(\delta^{\alpha}\right)$, where

$$
\omega_{p}(\delta, f)=\sup _{|t| \leq \delta}\|f(\cdot+t)-f(\cdot)\|_{p}
$$

is the integral modulus of continuity of $f \in L_{p}$.

\section{楚 Springer}

(C) 2015 Deǧer and Küçükaslan; licensee Springer. This is an Open Access article distributed under the terms of the Creative Commons Attribution License (http://creativecommons.org/licenses/by/4.0), which permits unrestricted use, distribution, and reproduction in any medium, provided the original work is properly credited. 
We shall also use the notations

$$
\Delta a_{n}=a_{n}-a_{n+1}, \quad \Delta_{m} a(n, m)=a(n, m)-a(n, m+1) .
$$

In this paper we are interested in the following two statements and will proceed in these directions.

1. One of the basic problems in the theory of approximations of functions and the theory of Fourier series is to examine the degree of approximation in given function spaces by certain methods. Naturally, there arises the question how we can generalize these approximation methods. The summability methods used in approximations belong to these methods. In this sense, we will give a generalization of deferred Cesáro means which includes Woronoi-Nörlund and Riesz methods as a summability method in Section 1. We know that the Nörlund and Riesz methods generalize the well-known Cesáro method which has an important place in this theory. In Section 4, we will establish some of summability properties related to this generalization.

2. As an application of these methods in theory of Fourier series, we will consider the degree of approximation in accordance with generalized deferred Cesáro means in a generalized Hölder metric in Section 2 and present some applications of the approach concerned with some sequence classes in Section 3.

Under the outlook given above, let us start with the notation of these generalizations. Accordingly, let $a=\left(a_{n}\right)$ and $b=\left(b_{n}\right)$ be sequences of nonnegative integers with conditions

$$
a_{n}<b_{n}, \quad n=1,2,3, \ldots
$$

and

$$
\lim _{n \rightarrow \infty} b_{n}=+\infty
$$

The deferred Cesáro mean, $(D)$ (see [1]), determined by $a$ and $b$ is defined as

$$
D_{n}=D_{a}^{b}=\frac{S_{a_{n}+1}+S_{a_{n}+2}+\cdots+S_{b_{n}}}{b_{n}-a_{n}}=\frac{1}{b_{n}-a_{n}} \sum_{k=a_{n}+1}^{b_{n}} S_{k},
$$

where $\left(S_{k}\right)$ is a sequence of real or complex numbers. Since each $D_{a}^{b}$ with conditions (1) and (2) satisfies the Silverman-Toeplitz conditions, every $D_{a}^{b}$ is regular. Note that $D_{a}^{b}$ involves, except in the case $a_{n}=0$ for all $n$, means of deferred elements of $\left(S_{n}\right)$. It is also well known that $D_{n-1}^{n}$ is the identity transformation and $D_{0}^{n}$ is the $(C, 1)$ transformation. The basic properties of $D_{a}^{b}$ can be found in [1]. By considering the deferred Cesáro means, we write the following notations with conditions (1) and (2). Let $\left(p_{n}\right)$ be a sequence of positive real numbers. Then we write

$$
D_{a}^{b} N_{n}(f ; x)=\frac{1}{P_{0}^{b_{n}-a_{n}-1}} \sum_{m=a_{n}+1}^{b_{n}} p_{b_{n}-m} S_{m}(f ; x)
$$

and

$$
D_{a}^{b} R_{n}(f ; x)=\frac{1}{P_{a_{n}+1}^{b_{n}}} \sum_{m=a_{n}+1}^{b_{n}} p_{m} S_{m}(f ; x),
$$


where

$$
P_{0}^{b_{n}-a_{n}-1}=\sum_{k=0}^{b_{n}-a_{n}-1} p_{k} \neq 0, \quad P_{a_{n}+1}^{b_{n}}=\sum_{k=a_{n}+1}^{b_{n}} p_{k} \neq 0,
$$

and

$$
S_{n}(f ; x)=\frac{1}{\pi} \int_{-\pi}^{\pi} f(x+t) D_{n}(t) d t
$$

in which

$$
D_{n}(t)=\frac{\sin \left(n+\frac{1}{2}\right) t}{2 \sin \left(\frac{t}{2}\right)}
$$

We will call these two methods the deferred Woronoi-Nörlund means, $\left(D_{a}^{b} N, p\right)$, and the deferred Riesz means, $\left(D_{a}^{b} R, p\right)$, respectively. In the case $b_{n}=n$ and $a_{n}=0$, the methods $D_{a}^{b} N_{n}(f ; x)$ and $D_{a}^{b} R_{n}(f ; x)$ give us the classically known Woronoi-Nörlund and Riesz means, respectively. Provided that $p_{n}=1$ for all $n(\geq 0)$, both of them yield the deferred Cesáro means

$$
D_{a}^{b}(f ; x)=\frac{1}{b_{n}-a_{n}} \sum_{m=a_{n}+1}^{b_{n}} S_{m}(f ; x)
$$

of $S_{m}(f, x)$. In addition to this, if $b_{n}=n, a_{n}=0$, and $p_{n}=1$ for these two methods, then they coincide with the Cesáro method $C_{1}$. In the event that $a_{n}=0,\left(b_{n}\right)$ is a strictly increasing sequence of positive integers with $b(0)=0$ and $p_{n}=1$, then they give us the Cesáro submethod which is obtained by deleting a set of rows from the Cesáro matrix (see [2-4]).

\section{Approximation by generalized deferred Cesáro means in generalized Hölder metric}

Let

$$
H_{\alpha}=\left\{f \in C_{2 \pi}:|f(x)-f(y)| \leq K|x-y|^{\alpha}\right\},
$$

where $0<\alpha \leq 1$ and $K$ is a positive constant, not necessarily the same at each occurrence. It is well known that $H_{\alpha}$ is a Banach space (see Prösdorff [5]) with the norm $\|\cdot\|_{\alpha}$ defined by

$$
\|f\|_{\alpha}=\|f\|_{C}+\sup _{x \neq y} \Delta^{\alpha} f(x, y),
$$

where

$$
\Delta^{\alpha} f(x, y)=\frac{|f(x)-f(y)|}{|x-y|^{\alpha}} \quad(x \neq y),
$$

by convention $\Delta^{0} f(x, y)=0$, and

$$
\|f\|_{C}=\sup _{x \in[-\pi, \pi]}|f(x)| .
$$


The metric generated by the norm (3) on $H_{\alpha}$ is called the Hölder metric. Prösdorff has studied the degree of approximation in the Hölder metric and proved the following theorem.

Theorem A [5] Let $f \in H_{\alpha}(0<\alpha \leq 1)$ and $0 \leq \beta<\alpha \leq 1$. Then

$$
\left\|\sigma_{n}(f)-f\right\|_{\beta}=O(1) \begin{cases}n^{\beta-\alpha}, & 0<\alpha<1 \\ n^{\beta-1} \ln n, & \alpha=1\end{cases}
$$

where $\sigma_{n}(f)$ is the Cesáro means of the Fourier series of $f$.

The case $\beta=0$ in Theorem $\mathrm{A}$ is due to Alexits [6]. Chandra obtained a generalization of Theorem A by considering the Woronoi-Nörlund transform [7]. Later, Mohapatra and Chandra considered the problem by a matrix means of the Fourier series of $f \in H_{\alpha}[8]$.

A generalization of the Hölder metric was given by Das et al. (see [9]). Accordingly, let

$$
H(\alpha, p):=\left\{f \in L^{p}, 0<p \leq \infty:\|f(x+h)-f(x)\|_{p}=O\left(|h|^{\alpha}\right)\right\}
$$

for $0<\alpha \leq 1 . H(\alpha, p)$ is a Banach space for $p \geq 1$ by the following norm:

$$
\begin{aligned}
& \|f\|_{(\alpha, p)}=\|f\|_{p}+\sup \frac{\|f(x+h)-f(x)\|_{p}}{|h|^{\alpha}}, \\
& \|f\|_{(0, p)}=\|f\|_{p} .
\end{aligned}
$$

In [9], one studied the results regarding the degree of approximation by an infinite matrix means involved in the deferred Cesaro means in a generalized Hölder metric. In the case $p=\infty$, the space $H(\alpha, \infty)$ coincides with the space $H_{\alpha}$ given by Prösdorff in [5].

In this section, we shall consider the degree of approximation of $f \in H(\alpha, p)$ with respect to the norm in the space $H(\alpha, p)$ by the deferred Woronoi-Nörlund means and the deferred Riesz means of the Fourier series of the function $f$ by taking into account the method in [9].

Theorem 2.1 Suppose that $\left(p_{n}\right)$ be a positive sequence with condition

$$
\sum_{k=a_{n}+1}^{b_{n}-1}\left|\Delta p_{k}\right|=O\left(\left|p_{b_{n}}-p_{a_{n}+1}\right|\right)
$$

If $f \in H(\alpha, p)$ for $p \geq 1$ and $0 \leq \beta<\alpha \leq 1$, then

$$
\left\|f-D_{a}^{b} R_{n}(f)\right\|_{(\beta, p)}=O(1) \begin{cases}\frac{\left\{1+\log \left(\frac{\left(b_{n}+1\right)}{\left(b_{n}-a_{n}\right)}\right)\right\}^{\beta / \alpha}}{\left.\left(b_{n}-a_{n}\right)\right)^{-\beta}}+\Phi_{p}(a, b)\left(b_{n}-a_{n}\right)^{1-\alpha+\beta}, & 0<\alpha<1 ; \\ \frac{\left\{1+\log \left(\frac{\left(b_{n}+1\right)}{\left(b_{n}-a_{n}\right)}\right)\right\}^{\beta}}{\left.\left(b_{n}-a_{n}\right)\right)^{-\beta}}+\frac{\Phi_{p}(a, b)\left(b_{n}-a_{n}\right)^{\beta}}{\left(\log \left(b_{n}-a_{n}\right)\right)^{\beta-1}}, & \alpha=1,\end{cases}
$$

where

$$
\Phi_{p}(a, b)=\frac{1}{P_{a_{n}+1}^{b_{n}}}\left\{\left|p_{b_{n}}-p_{a_{n}+1}\right|+p_{b_{n}}+p_{a_{n}+1}\right\} .
$$


Proof A standard computation shows that

$$
S_{n}(f, x)-f(x)=\frac{1}{2 \pi} \int_{0}^{\pi} \Psi_{x}(t)\left(\frac{\sin \left(n+\frac{1}{2}\right) t}{\sin \left(\frac{t}{2}\right)}\right) d t
$$

where $\Psi_{x}(t):=f(x+t)+f(x-t)-2 f(x)$. Taking into account (6) and the deferred Riesz mean of $S_{n}(f ; x)$, we write

$$
\begin{aligned}
l_{n}(x) & :=D_{a}^{b} R_{n}(f ; x)-f(x)=\frac{1}{P_{a_{n}+1}^{b_{n}}} \sum_{m=a_{n}+1}^{b_{n}} p_{m}\left(S_{m}(f ; x)-f(x)\right) \\
& =\int_{0}^{\pi} \Psi_{x}(t) \frac{1}{\pi P_{a_{n}+1}^{b_{n}}} \sum_{m=a_{n}+1}^{b_{n}} p_{m} D_{m}(t) d t=\int_{0}^{\pi} \Psi_{x}(t) K_{n}(t) d t,
\end{aligned}
$$

where

$$
K_{n}(t):=\frac{1}{\pi P_{a_{n}+1}^{b_{n}}} \sum_{m=a_{n}+1}^{b_{n}} p_{m} D_{m}(t) .
$$

We first note that

$$
\sum_{m=a_{n}+1}^{b_{n}} p_{m} \sin \left(m+\frac{1}{2}\right) t=O\left(\frac{1}{t}\right)\left\{\sum_{m=a_{n}+1}^{b_{n}-1}\left|\Delta_{m} p_{m}\right|+p_{a_{n}+1}+p_{b_{n}}\right\}
$$

by using Abel?s transformation and the Jordan inequality. Furthermore, taking into account the definition of $D_{a}^{b} R_{n}(f ; x)$, we see that

$$
\left|K_{n}(t)\right|=O\left(\frac{t^{-1}}{P_{a_{n}+1}^{b_{n}}}\right) \sum_{m=a_{n}+1}^{b_{n}}\left|p_{m}\right|(m+1) t=O\left(b_{n}+1\right)
$$

and

$$
K_{n}(t)=O\left(t^{-1}\right)
$$

for all $0<t \leq \pi$. An elementary calculation gives

$$
\begin{aligned}
l_{n}(x+y)-l_{n}(x) \\
\quad=\int_{0}^{\pi}\left(\Psi_{x+y}(t)-\Psi_{x}(t)\right) K_{n}(t) d t \\
=\int_{0}^{\pi}\{f(x+y+t)-f(x+t)+f(x+y-t)-f(x-t)-2(f(x+y)-f(x))\} K_{n}(t) d t \\
=\int_{-\pi}^{\pi}(f(x+y+t)-f(x+t)) K_{n}(t) d t-2 \int_{0}^{\pi}(f(x+y)-f(x)) K_{n}(t) d t .
\end{aligned}
$$


Accordingly, we have

$$
\begin{aligned}
\left\|l_{n}(\cdot+y)-l_{n}(\cdot)\right\|_{p} \leq & \left\{\frac{1}{2 \pi} \int_{0}^{2 \pi}\left|\int_{-\pi}^{\pi}(f(x+t+y)-f(x+t)) K_{n}(t) d t\right|^{p} d x\right\}^{1 / p} \\
& +\left\{\frac{1}{2 \pi} \int_{0}^{2 \pi}\left|2 \int_{0}^{\pi}(f(x+y)-f(x)) K_{n}(t) d t\right|^{p} d x\right\}^{1 / p} \\
\leq & \int_{-\pi}^{\pi}\left|K_{n}(t)\right|\left\{\frac{1}{2 \pi} \int_{0}^{2 \pi}|f(x+t+y)-f(x+t)|^{p} d x\right\}^{1 / p} d t \\
& +2 \int_{0}^{\pi}\left|K_{n}(t)\right|\left\{\frac{1}{2 \pi} \int_{0}^{2 \pi}|f(x+y)-f(x)|^{p} d x\right\}^{1 / p} d t \\
= & O\left(|y|^{\alpha}\right) \int_{0}^{\pi}\left|K_{n}(t)\right| d t=: I
\end{aligned}
$$

by considering $f \in H(\alpha, p)$ and the general form of the Minkowski inequality. Divide the integral $I$ into three parts:

$$
I=O\left(|y|^{\alpha}\right)\left(\int_{0}^{1 /\left(b_{n}+1\right)}+\int_{1 /\left(b_{n}+1\right)}^{1 /\left(b_{n}-a_{n}\right)}+\int_{1 /\left(b_{n}-a_{n}\right)}^{\pi}\right)\left|K_{n}(t)\right| d t=: I_{1}+I_{2}+I_{3} .
$$

These integrals, by (8) and (9), can easily be estimated by standard methods:

$$
\begin{aligned}
I_{1} & =O\left(|y|^{\alpha}\left(b_{n}+1\right)\right) \int_{0}^{1 /\left(b_{n}+1\right)} d t=O\left(|y|^{\alpha}\right), \\
I_{2} & =O\left(|y|^{\alpha}\right) \int_{1 /\left(b_{n}+1\right)}^{1 /\left(b_{n}-a_{n}\right)}\left|K_{n}(t)\right| d t \\
& =O\left(|y|^{\alpha}\right) \int_{1 /\left(b_{n}+1\right)}^{1 /\left(b_{n}-a_{n}\right)} \frac{d t}{t}=O\left(|y|^{\alpha} \log \frac{b_{n}+1}{b_{n}-a_{n}}\right)
\end{aligned}
$$

and finally by (4) and (7) we have

$$
\begin{aligned}
I_{3} & =|y|^{\alpha} \int_{1 /\left(b_{n}-a_{n}\right)}^{\pi}\left|K_{n}(t)\right| d t=O\left(|y|^{\alpha} \Phi_{p}(a, b)\right) \int_{1 /\left(b_{n}-a_{n}\right)}^{\pi} \frac{d t}{t^{2}} \\
& =O\left(|y|^{\alpha} \Phi_{p}(a, b)\left(b_{n}-a_{n}\right)\right) .
\end{aligned}
$$

Therefore, collecting our estimates we obtain

$$
J_{1}:=I_{1}+I_{2}=O\left(|y|^{\alpha}\left(1+\log \frac{b_{n}+1}{b_{n}-a_{n}}\right)\right)
$$

and

$$
J_{2}:=I_{3}=O\left(|y|^{\alpha} \Phi_{p}(a, b)\left(b_{n}-a_{n}\right)\right) .
$$

We need another estimation of the order of $\left\|l_{n}(\cdot+y)-l_{n}(\cdot)\right\|_{p}$, as different from above, to reach the required result. For this aim, we will proceed in the following way to get another 
one. We write

$$
\begin{aligned}
\left\|l_{n}(\cdot+y)-l_{n}(\cdot)\right\|_{p} \leq & O(1)\left\{\int_{0}^{\pi}\|f(\cdot+t)-f(\cdot)\|_{p}\left|K_{n}(t)\right| d t\right. \\
& \left.+\int_{0}^{\pi}\|f(\cdot-t)-f(\cdot)\|_{p}\left|K_{n}(t)\right| d t\right\} \\
= & O(1) \int_{0}^{\pi} t^{\alpha}\left|K_{n}(t)\right| d t:=J
\end{aligned}
$$

by using $\|f(\cdot+t)-f(\cdot)\|_{p}=O\left(|t|^{\alpha}\right)$ and the generalized Minkowski inequality. Let us split the integral $J$ into two parts:

$$
J=\left(\int_{0}^{1 /\left(b_{n}-a_{n}\right)}+\int_{1 /\left(b_{n}-a_{n}\right)}^{\pi}\right) t^{\alpha}\left|K_{n}(t)\right| d t=: J_{1}+J_{2} .
$$

$J_{1}$ and $J_{2}$ can easily be estimated for any $\alpha \leq 1$ as follows. According to (9),

$$
J_{1}=O(1) \int_{0}^{1 /\left(b_{n}-a_{n}\right)} t^{\alpha-1} d t=O\left(\frac{1}{\left(b_{n}-a_{n}\right)^{\alpha}}\right)
$$

and we have, by (4) and (7),

$$
\begin{aligned}
J_{2} & =O\left(\Phi_{p}(a, b)\right) \int_{1 /\left(b_{n}-a_{n}\right)}^{\pi} t^{\alpha-2} d t \\
& =O\left(\Phi_{p}(a, b)\right) \begin{cases}\left(b_{n}-a_{n}\right)^{1-\alpha}, & 0<\alpha<1 ; \\
\log \left(b_{n}-a_{n}\right), & \alpha=1 .\end{cases}
\end{aligned}
$$

Combining the integral estimates (11)-(14) in the form $J_{k}=J_{k}^{\beta / \alpha} J_{k}^{1-\beta / \alpha}$ for $k=1,2$, we observe that

$$
J_{1}=O\left(|y|^{\beta}\right)\left(1+\log \frac{b_{n}+1}{b_{n}-a_{n}}\right)^{\beta / \alpha}\left(b_{n}-a_{n}\right)^{\beta-\alpha}
$$

and

$$
\begin{aligned}
J_{2} & =O\left(|y|^{\beta}\right) \Phi_{p}(a, b)\left(b_{n}-a_{n}\right)^{\beta / \alpha} \begin{cases}\left(\left(b_{n}-a_{n}\right)^{1-\alpha}\right)^{1-\beta / \alpha}, & 0<\alpha<1 ; \\
\left(\log \left(b_{n}-a_{n}\right)\right)^{1-\beta}, & \alpha=1,\end{cases} \\
& =O\left(|y|^{\beta}\right) \Phi_{p}(a, b) \begin{cases}\left(b_{n}-a_{n}\right)^{1+\beta-\alpha}, & 0<\alpha<1 ; \\
\left(b_{n}-a_{n}\right)^{\beta}\left(\log \left(b_{n}-a_{n}\right)\right)^{1-\beta}, & \alpha=1 .\end{cases}
\end{aligned}
$$

Therefore, by considering the previous evaluations, we have

$$
\begin{aligned}
\sup _{y \neq 0} \frac{\left\|l_{n}(\cdot+y)-l_{n}(\cdot)\right\|_{p}}{|y|^{\beta}}= & O(1)\left(1+\log \frac{b_{n}+1}{b_{n}-a_{n}}\right)^{\beta / \alpha}\left(b_{n}-a_{n}\right)^{\beta-\alpha} \\
& +O(1) \Phi_{p}(a, b) \begin{cases}\left(b_{n}-a_{n}\right)^{1+\beta-\alpha}, & 0<\alpha<1 ; \\
\left(b_{n}-a_{n}\right)^{\beta}\left(\log \left(b_{n}-a_{n}\right)\right)^{1-\beta}, & \alpha=1 .\end{cases}
\end{aligned}
$$


Furthermore, following the same considerations as in the estimation of (13)-(14) we get

$$
\left\|D_{a}^{b} R_{n}(f)-f\right\|_{p}=O\left(\frac{1}{\left(b_{n}-a_{n}\right)^{\alpha}}\right)+O\left(\Phi_{p}(a, b)\right) \begin{cases}\left(b_{n}-a_{n}\right)^{1-\alpha}, & 0<\alpha<1 \\ \log \left(b_{n}-a_{n}\right), & \alpha=1\end{cases}
$$

Collecting our partial results, (5) is obtained and this completes the proof.

If $\left(p_{n}\right)$ is nonincreasing, then it is obvious that the condition (4) is also true. That is, in the case $\left(p_{n}\right)$ is nonincreasing,

$$
\sum_{k=a_{n}+1}^{b_{n}-1}\left|\Delta p_{k}\right|=\sum_{k=a_{n}+1}^{b_{n}-1}\left\{p_{k}-p_{k+1}\right\}=-p_{b_{n}}+p_{a_{n}+1}
$$

and

$$
\Phi_{p}(a, b)=\frac{O\left(p_{a_{n}+1}\right)}{P_{a_{n}+1}^{b_{n}}}
$$

Therefore we can write the following corollary of Theorem 2.1.

Corollary 2.2 Let $\left(p_{n}\right)$ be a positive and nonincreasing sequence. If $\in H(\alpha, p)$ for $p \geq 1$ and $0 \leq \beta<\alpha \leq 1$, then

$$
\left\|f-D_{a}^{b} R_{n}(f)\right\|_{(\beta, p)}=O(1) \begin{cases}\frac{\left\{1+\log \left(\frac{\left(b_{n}+1\right)}{\left(b_{n}-a_{n}\right)}\right)\right\}^{\beta / \alpha}}{\left(b_{n}-a_{n}\right)^{\alpha-\beta}}+\frac{p_{a_{n}+1}}{P_{a_{n}+1}^{b_{n}}}\left(b_{n}-a_{n}\right)^{1-\alpha+\beta}, & 0<\alpha<1 ; \\ \frac{\left\{1+\log \left(\frac{\left(b_{n}+1\right)}{\left(b_{n}-a_{n}\right)}\right)\right\}^{\beta}}{\left(b_{n}-a_{n}\right)^{1-\beta}}+\frac{p_{a_{n}+1}}{P_{a_{n}+1}^{b_{n}}} \frac{\left(b_{n}-a_{n}\right)^{\beta}}{\left(\log \left(b_{n}-a_{n}\right)\right)^{\beta-1}}, & \alpha=1 .\end{cases}
$$

Similarly, if $\left(p_{n}\right)$ is nondecreasing, then

$$
\sum_{k=a_{n}+1}^{b_{n}-1}\left|\Delta p_{k}\right|=\sum_{k=a_{n}+1}^{b_{n}-1}\left\{p_{k+1}-p_{k}\right\}=p_{b_{n}}-p_{a_{n}+1}
$$

and

$$
\Phi_{p}(a, b)=\frac{O\left(p_{b_{n}}\right)}{P_{a_{n}+1}^{b_{n}}}
$$

Taking into account this fact, we write the next result as another consequence of Theorem 2.1 .

Corollary 2.3 Let $\left(p_{n}\right)$ be a positive and nondecreasing sequence. If $\in \in H(\alpha, p)$ for $p \geq 1$ and $0 \leq \beta<\alpha \leq 1$, then

$$
\left\|f-D_{a}^{b} R_{n}(f)\right\|_{(\beta, p)}=O(1) \begin{cases}\frac{\left\{1+\log \left(\frac{\left(b_{n}+1\right)}{\left(b_{n}-a_{n}\right)}\right)\right\}^{\beta / \alpha}}{\left(b_{n}-a_{n}\right)^{-\beta}}+\frac{p_{b_{n}}}{P_{a_{n}+1}^{b_{n}}}\left(b_{n}-a_{n}\right)^{1-\alpha+\beta}, & 0<\alpha<1 ; \\ \frac{\left\{1+\log \left(\frac{\left(b_{n}+1\right)}{\left(b_{n}-a_{n}\right)}\right)\right\}^{\beta}}{\left(b_{n}-a_{n}\right)^{1-\beta}}+\frac{p_{b_{n}}}{P_{a_{n}+1}^{b_{n}}} \frac{\left(b_{n}-a_{n}\right)^{\beta}}{\left(\log \left(b_{n}-a_{n}\right)\right)^{\beta-1}}, & \alpha=1 .\end{cases}
$$

The next result is related to the deferred Woronoi-Nörlund means in a generalized Hölder metric. 
Theorem 2.4 Let $\left(p_{n}\right)$ be a positive sequence such that the condition

$$
\sum_{k=a_{n}+1}^{b_{n}-1}\left|\Delta_{k} p_{b_{n}-k}\right|=O\left(\left|p_{b_{n}-a_{n}-1}-p_{0}\right|\right)
$$

holds. If $\in H(\alpha, p)$ for $p \geq 1$ and $0 \leq \beta<\alpha \leq 1$, then

$$
\left\|f-D_{a}^{b} N_{n}(f)\right\|_{(\beta, p)}=O(1) \begin{cases}\frac{\left\{1+\log \left(\frac{\left(b_{n}+1\right)}{\left(b_{n}-a_{n}\right)}\right)\right\}^{\beta / \alpha}}{\left(b_{n}-a_{n}\right)^{\alpha-\beta}}+\Theta_{p}(a, b)\left(b_{n}-a_{n}\right)^{1-\alpha+\beta}, & 0<\alpha<1 ; \\ \frac{\left\{1+\log \left(\frac{\left.b_{n}+1\right)}{\left(b_{n}-a_{n}\right)}\right)\right\}^{\beta}}{\left(b_{n}-a_{n}\right)^{1-\beta}}+\frac{\Theta_{p}(a, b)\left(b_{n}-a_{n}\right)^{\beta}}{\left(\log \left(b_{n}-a_{n}\right)\right)^{\beta-1}}, & \alpha=1,\end{cases}
$$

where

$$
\Theta_{p}(a, b)=\frac{1}{P_{0}^{b_{n}-a_{n}-1}}\left\{\left|p_{b_{n}-a_{n}-1}-p_{0}\right|+p_{b_{n}-a_{n}-1}+p_{0}\right\} .
$$

Proof First of all we need the following estimations given in Theorem 2.1. By considering (6) and the deferred Woronoi-Nörlund means of $S_{n}(f ; x)$, we write

$$
\begin{aligned}
D_{a}^{b} N_{n}(f ; x)-f(x) & =\frac{1}{P_{0}^{b_{n}-a_{n}-1}} \sum_{m=a_{n}+1}^{b_{n}} p_{b_{n}-m}\left(S_{m}(f ; x)-f(x)\right) \\
& =\int_{0}^{\pi} \Psi_{x}(t) \frac{1}{\pi P_{0}^{b_{n}-a_{n}-1}} \sum_{m=a_{n}+1}^{b_{n}} p_{b_{n}-m} \frac{\sin \left(m+\frac{1}{2}\right) t}{2 \sin \left(\frac{t}{2}\right)} d t \\
& =\int_{0}^{\pi} \Psi_{x}(t) B_{n}(t) d t,
\end{aligned}
$$

where

$$
B_{n}(t):=\frac{1}{\pi P_{0}^{b_{n}-a_{n}-1}} \sum_{m=a_{n}+1}^{b_{n}} p_{b_{n}-m} \frac{\sin \left(m+\frac{1}{2}\right) t}{2 \sin \left(\frac{t}{2}\right)} .
$$

Moreover, we get

$$
\sum_{m=a_{n}+1}^{b_{n}} p_{b_{n}-m} \sin \left(m+\frac{1}{2}\right) t=O\left(\frac{1}{t}\right)\left\{\sum_{m=a_{n}+1}^{b_{n}-1}\left|\Delta_{m} p_{b_{n}-m}\right|+p_{b_{n}-a_{n}-1}+p_{0}\right\}
$$

by using Abel?s transformation and the Jordan inequality. Ultimately, an elementary calculation also gives us

$$
\left|B_{n}(t)\right|=O\left(\frac{t^{-1}}{P_{0}^{b_{n}-a_{n}-1}}\right) \sum_{m=a_{n}+1}^{b_{n}} p_{b_{n}-m}(m+1) t=O\left(b_{n}+1\right)
$$

and

$$
B_{n}(t)=O\left(t^{-1}\right)
$$

for all $0<t \leq \pi$. After this, the proof runs along the same lines as that of Theorem 2.1. 
Analogous results to Corollary 2.2 and Corollary 2.3 can also be given for the deferred Nörlund means. Moreover, since $D_{0}^{n} R_{n}$ and $D_{0}^{n} N_{n}$ in the case $p_{n}=1$ (for all $n$ ) coincide with the Cesáro method $C_{1}$, Theorem 2.1 and Theorem 2.4 are reduced to the result of Prösdorff in $H(\alpha, \infty)$ space. Furthermore we know that if $p_{n}=1$ for all $n$, then these two methods give us the deferred Cesáro means. Therefore our results in Theorem 2.1 and Theorem 2.4 coincide with the results relevant to the deferred Cesáro means of Das et al. (see [9]) in some cases.

\section{Applications related to some sequence classes}

While taking into account these deferred methods, the monotonicity conditions on the sequence $\left(p_{n}\right)$ are important. Especially, the sum of the left side in (4) and (15) in Theorem 2.1 and Theorem 2.4 takes over in the case we have some sequence classes as given the following, respectively. So, let us recall the definitions of some classes of numerical sequences. Some details related to these classes can be found in [10] and [11]. Let $u:=\left(u_{n}\right)$ be a nonnegative sequence.

A sequence $u$ is called almost monotone decreasing (briefly $u \in A M D S$ ) (increasing (briefly $u \in A M I S)$ ), if there exists a constant $K:=K(u)$ which only depends on $u$ such that

$$
u_{n} \leq K u_{m} \quad\left(u_{m} \leq K u_{n}\right)
$$

for all $n \geq m$. This notion is due to Bernstein [12].

A sequence $u$ is called a head bounded variation sequence (briefly $u \in H B V S$ ), if it has the property

$$
\sum_{m=0}^{k-1}\left|\Delta u_{m}\right| \leq K(u) u_{k}
$$

for all natural numbers $k$, or only for all $k \leq N$ if the sequence $u$ has only finite nonzero terms and the last nonzero term $u_{N}$.

A sequence $u$ tending to zero is called a rest bounded variation sequence (briefly $u \in$ $R B V S)$, if it has the property

$$
\sum_{m=k}^{\infty}\left|\Delta u_{m}\right| \leq K(u) u_{k}
$$

for all natural numbers $k$ (see [10]). It is clear that the following inclusions are true for the above classes of numerical sequences:

$$
N I S \subset R B V S \subset A M D S, \quad N D S \subset H B V S \subset A M I S,
$$

where NIS and NDS denote the classes of numerical sequences nonincreasing and nondecreasing, respectively. Taking into account these inclusions, we will revise the results given in Section 2 by weakening the monotonicity conditions. 
Theorem 3.1 Let $\left(p_{n}\right)$ be a positive sequence with condition

$$
\text { (i) } \sum_{k=a_{n}+1}^{b_{n}-1}\left|\Delta p_{k}\right|=O\left(p_{a_{n}+1}\right) \quad \text { or } \quad \text { (ii) } \sum_{k=a_{n}+1}^{b_{n}-1}\left|\Delta p_{k}\right|=O\left(p_{b_{n}}\right) \text {. }
$$

If $f \in H(\alpha, p)$ for $p \geq 1$ and $0 \leq \beta<\alpha \leq 1$, then (5) holds with $\Phi_{p}(a, b)=O(1)\left\{p_{b_{n}}+\right.$ $\left.p_{a_{n}+1}\right\} / P_{a_{n}+1}^{b_{n}}$.

We note that if $\left(p_{n}\right) \in N I S$ (or NDS), then (17)(i) (or (ii)) holds. In this case, Theorem 3.1 is reduced to Corollary 2.2 (Corollary 2.3).

If $\left(p_{n}\right) \in R B V S$ (or $H B V S$ ), then it is obvious that the condition (17)(i) (or (ii)) is also true. Therefore, we write the following result as a consequence of Theorem 3.1.

Corollary 3.2 Let $p \geq 1$ and $0 \leq \beta<\alpha \leq 1$. If $\left(p_{n}\right)$ belongs to RBVS (or HBVS), then for $f \in H(\alpha, p)$, (5) holds with $\Phi_{p}(a, b)=O(1)\left\{p_{b_{n}}+p_{a_{n}+1}\right\} / P_{a_{n}+1}^{b_{n}}$.

Let us consider the family $\left\{\left(u_{n}^{(k)}\right)\right\}_{1}^{\infty}$ of positive sequences such that

$$
\left(u_{n}^{(k)}\right) \in A M I S(A M D S)
$$

for $k=1,2, \ldots$. Then from the definition of $A M I S(A M D S)$, there exists a constant $C_{k}:=$ $C\left(u_{n}^{(k)}\right)$ which only depends on $\left(u_{n}^{(k)}\right)$ such that

$$
C_{k} u_{n}^{(k)} \geq u_{m}^{(k)} \quad\left(C_{k} u_{m}^{(k)} \geq u_{n}^{(k)}\right)
$$

for all $n \geq m$ and $k=1,2, \ldots$. We want to build a subclass of numerical sequences that belongs to AMIS (AMDS) to satisfy our aim. According to this, we define

$$
\begin{aligned}
& A M I S^{+}=\left\{u_{n}^{(k)}: 0<C_{k}<\pi,\left(u_{n}^{(k)}\right) \in A M I S, k=1,2, \ldots\right\} \\
& \left(A M D S^{+}=\left\{u_{n}^{(k)}: 0<C_{k}<\pi,\left(u_{n}^{(k)}\right) \in A M D S, k=1,2, \ldots\right\}\right) .
\end{aligned}
$$

It is easy to see that $N D S \subset A M I S^{+}\left(N I S \subset A M D S^{+}\right)$when taking into account $C_{k}=1$ for all $k$. Therefore if $\left(p_{n}\right) \in A M I S^{+}\left(A M D S^{+}\right)$, then there exists a number $\kappa>0\left(\kappa_{1}>0\right)$ such that

$$
1=\frac{1}{P_{a_{n}+1}^{b_{n}}} \sum_{k=a_{n}+1}^{b_{n}} p_{k} \leq \kappa\left(b_{n}-a_{n}\right) \frac{p_{b_{n}}}{P_{a_{n}+1}^{b_{n}}}\left(\kappa\left(b_{n}-a_{n}\right) \frac{p_{a_{n}+1}}{P_{a_{n}+1}^{b_{n}}}\right)
$$

holds. Then if we take $\frac{\kappa p_{b_{n}}}{P_{a_{n}+1}^{b_{n}}}\left(\frac{\kappa 1 p_{a_{n}+1}}{P_{a_{n}+1}^{b_{n}}}\right)$ instead of $\frac{1}{b_{n}-a_{n}}$ in (10), we can write the following result, which weakens the condition of monotonicity on Corollary 2.3 (Corollary 2.2).

Corollary 3.3 Under the conditions of Theorem 3.1, we have

$$
\begin{aligned}
\| f & -D_{a}^{b} R_{n}(f) \|_{(\beta, p)} \\
& =O(1) \begin{cases}\left(\Lambda_{\left(p_{n}\right)}\right)^{\alpha-\beta}\left\{\left\{1+\log \left(\left(b_{n}+1\right) \Lambda_{\left(p_{n}\right)}\right)\right\}^{\beta / \alpha}+1\right\}, & \alpha \in(0,1) ; \\
\left(\Lambda_{\left(p_{n}\right)}\right)^{1-\beta}\left\{\left\{1+\log \left(\left(b_{n}+1\right) \Lambda_{\left(p_{n}\right)}\right)\right\}^{\beta}+\frac{1}{\left(\log \left(\frac{1}{\left.\Lambda_{\left(p_{n}\right)}\right)}\right)\right.}\right\}, & \alpha=1,\end{cases}
\end{aligned}
$$


where

$$
\Lambda_{\left(p_{n}\right)}= \begin{cases}\kappa \frac{p_{b_{n}}}{P_{a_{n}+1}^{b_{n}},}, & \left(p_{n}\right) \in A M I S^{+} \\ \kappa_{1} \frac{p_{a_{n}+1}}{P_{a_{n}+1}^{b_{n}}}, & \left(p_{n}\right) \in A M D S^{+}\end{cases}
$$

Similarly to the above considerations, the deferred Woronoi-Nörlund means can be written as follows.

Theorem 3.4 Let $\left(p_{n}\right)$ be a positive sequence with condition

(i) $\sum_{k=a_{n}+1}^{b_{n}-1}\left|\Delta p_{b_{n}-k}\right|=O\left(p_{b_{n}-a_{n}-1}\right) \quad$ or $\quad$ (ii) $\sum_{k=a_{n}+1}^{b_{n}-1}\left|\Delta p_{b_{n}-k}\right|=O\left(p_{0}\right)$.

If $f \in H(\alpha, p)$ for $p \geq 1$ and $0 \leq \beta<\alpha \leq 1$, then (16) holds with $\Theta_{p}(a, b)=O(1)\left\{p_{b_{n}-a_{n}-1}+\right.$ $\left.p_{0}\right\} / P_{0}^{b_{n}-a_{n}-1}$.

Also it is possible to give similar results to the above in the event that $\left(p_{n}\right)$ belongs to NDS (NIS) and HBVS (RBVS). But we will only give the next result related to $\left(p_{n}\right) \in$ $A M I S^{+}\left(A M D S^{+}\right)$without further details. In the case $\left(p_{n}\right) \in A M I S^{+}\left(A M D S^{+}\right)$, there exists a number $\delta>0\left(\delta_{1}>0\right)$ such that

$$
1=\frac{1}{P_{0}^{b_{n}-a_{n}-1}} \sum_{k=a_{n}+1}^{b_{n}} p_{b_{n}-a_{n}-1} \leq \delta\left(b_{n}-a_{n}\right) \frac{p_{b_{n}-a_{n}-1}}{P_{0}^{b_{n}-a_{n}-1}}\left(\delta_{1}\left(b_{n}-a_{n}\right) \frac{p_{0}}{P_{0}^{b_{n}-a_{n}-1}}\right) .
$$

Hence, by choosing $\frac{\delta p_{b_{n}-a_{n}-1}}{P_{0}^{b_{n}-a_{n}-1}}\left(\frac{\delta 1 p_{0}}{P_{0}^{b_{0}-a_{n}-1}}\right)$ in replacement of $\frac{1}{b_{n}-a_{n}}$ in (10), the subsequent result is as follows.

Corollary 3.5 Under the conditions of Theorem 3.4, we get

$$
\begin{aligned}
\| f & -D_{a}^{b} N_{n}(f) \|_{(\beta, p)} \\
& =O(1) \begin{cases}\left(\Upsilon_{\left(p_{n}\right)}\right)^{\alpha-\beta}\left\{\left\{1+\log \left(\left(b_{n}+1\right) \Upsilon_{\left(p_{n}\right)}\right)\right\}^{\beta / \alpha}+1\right\}, & 0<\alpha<1 ; \\
\left(\Upsilon_{\left(p_{n}\right)}\right)^{1-\beta}\left\{\left\{1+\log \left(\left(b_{n}+1\right) \Upsilon_{\left(p_{n}\right)}\right)\right\}^{\beta}+\frac{1}{\left(\log \left(\frac{1}{\Upsilon_{\left(p_{n}\right)}}\right) \beta^{\beta-1}\right.}\right\}, & \alpha=1,\end{cases}
\end{aligned}
$$

where

$$
\Upsilon_{\left(p_{n}\right)}= \begin{cases}\frac{\delta p_{b_{n}-a_{n}-1}}{P_{0}^{b_{n}-a_{n}-1},} & \left(p_{n}\right) \in A M I S^{+} \\ \frac{\delta_{1} p_{0}}{P_{0}^{b_{n}-a_{n}-1},} & \left(p_{n}\right) \in A M D S^{+}\end{cases}
$$

\section{Results on generalized deferred Cesáro means}

In this section, taking into account the generalized deferred Cesáro means under the perspective of the results of Agnew, we will discuss some results in connection with [1]. Before giving some results, let us recall some notations and definitions. Let $x=\left(x_{k}\right)$ be a sequence of real numbers and $U:=\left(d_{n, k}\right)$ a summability matrix. Then $U x$ is the sequence whose $n$th term is given by $\sigma_{n}=\sum_{k=1}^{\infty} d_{n, k} x_{k}$. The matrix $U$ is regular if $\lim _{n} \sigma_{n}=p$ whenever $\lim _{n} x_{n}=p$. The well-known Silverman-Toeplitz, $(S T)$, conditions characterize the 
regular matrices. According to this, the matrix $U$ is regular if and only if it satisfies the following three conditions:

$$
\begin{aligned}
& \lim _{n} d_{n, k}=0 \quad \text { for each } k=1,2,3, \ldots ; \\
& \sup _{n} \sum_{k=1}^{\infty}\left|d_{n, k}\right|<\infty \\
& \lim _{n} \sum_{k=1}^{\infty} d_{n, k}=1 .
\end{aligned}
$$

Throughout this part we are concerned with the transformations satisfying ST conditions. The Woronoi-Nörlund transformation, $(N, p)$, and the Riesz transformation, $(R, p)$, are obtained by taking $d_{n, k}=p_{n-k} / P_{n}$ and $d_{n, k}=p_{k} / P_{n}, n=0,1, \ldots, k=0,1, \ldots, n$ in $\sigma_{n}$ where $\left(p_{n}\right)$ is a given sequence of positive numbers such that $P_{n}=\sum_{k=0}^{n} p_{k} \neq 0, P_{-1}=p_{-1}=0$, respectively. Since the $(N, p)$ and $(R, p)$ transformations satisfy the $S T$ conditions, they are regular. Moreover, if we consider $d_{n, k}=p_{b_{n}-k} / P_{0}^{b_{n}-a_{n}-1}$ and $d_{n, k}=p_{k} / P_{a_{n}+1}^{b_{n}}, a_{n}<k \leq b_{n}$, then the deferred Woronoi-Nörlund and the deferred Riesz transformation are obtained, respectively. When considering the $\left(D_{a}^{b} N, p\right)$ and $\left(D_{a}^{b} R, p\right)$ transformations, since they satisfy the $S T$-conditions with (1) and (2), they are also regular.

If $\left(a_{n}\right)$ and $\left(b_{n}\right)$ satisfy, in addition to (1) and (2), the condition

$$
\frac{a_{n}}{b_{n}-a_{n}}=O(1)
$$

for all $n$, then $(D)$ is properly deferred; such a transformation is called a proper $(D)$ (see [1]). In this part, we will give a condition similar to (21) for a deferred Riesz transformation. Assume that $\left(a_{n}\right)$ and $\left(b_{n}\right)$ satisfy (1) and (2). Accordingly, if the condition

$$
\frac{p_{1}+p_{2}+\cdots+p_{a_{n}}}{P_{a_{n}+1}^{b_{n}}}=O(1)
$$

holds for $\left(p_{n}\right)$, then we shall say that $\left(D_{a}^{b} R, p\right)$ is properly deferred and such a transformation is called a proper $\left(D_{a}^{b} R, p\right)$. We see that if $\left(p_{n}\right)=1$ for all $n$ with the conditions (1) and (2), then $\left(D_{a}^{b} R, p\right)$ and the condition (22) are reduced to $(D)$ and the condition (21), respectively.

Theorem 4.1 $(R, p) \subset\left(D_{a}^{b} R, p\right)$ if and only if $\left(D_{a}^{b} R, p\right)$ is proper.

Proof Let $\left(S_{n}\right)$ be a sequence of real numbers. Then for any $\left(D_{a}^{b} R, p\right)$ transformation of $\left(S_{n}\right)$, we have

$$
\begin{aligned}
D_{a}^{b} R_{n} & =\frac{1}{P_{a_{n}+1}^{b_{n}}} \sum_{m=a_{n}+1}^{b_{n}} p_{m} S_{m} \\
& =\frac{p_{1}+p_{2}+\cdots+p_{b_{n}}}{P_{a_{n}+1}^{b_{n}}} R_{b_{n}}-\frac{p_{1}+p_{2}+\cdots+p_{a_{n}}}{P_{a_{n}+1}^{b_{n}}} R_{a_{n}} .
\end{aligned}
$$

Suppose that the relation (23) is considered as a transformation of the form $\sigma_{n}$ which carries $\left(R_{n}\right)$ into $\left(D_{a}^{b} R_{n}\right)$ and let $(R, p) \subset\left(D_{a}^{b} R, p\right)$. It is seen that (23) provides the conditions 
(18) and (20), since the Riesz transformation is regular. In order that (23) satisfies the condition (19),

$$
\frac{p_{1}+p_{2}+\cdots+p_{b_{n}}}{P_{a_{n}+1}^{b_{n}}}+\frac{p_{1}+p_{2}+\cdots+p_{a_{n}}}{P_{a_{n}+1}^{b_{n}}}=O(1)
$$

is necessary and sufficient, i.e. (22) is a necessary and sufficient condition. Therefore, $\left(D_{a}^{b} R, p\right)$ is proper. On the other hand, assume that $\left(D_{a}^{b} R, p\right)$ is proper. Then we know that (23) satisfies the $S T$ conditions. Moreover, since $(R, p)$ has an inverse, (23) is regular if and only if $(R, p) \subset\left(D_{a}^{b} R, p\right)$. Hence the proof is completed.

The next result is associated with the fact that Riesz transformations contain deferred Riesz transformations in which case we have the following.

Theorem $4.2\left(D_{a}^{n} R, p\right) \subset(R, p)$.

Proof Assume that the sequence $\left(S_{n}\right)$ is $\left(D_{a}^{n} R, p\right)$-summable to the sum $u$, i.e.

$$
\lim _{n \rightarrow \infty} D_{a}^{b} R_{n}=u
$$

We write

$$
\begin{aligned}
R_{n}= & \frac{1}{P_{n}}\left\{p_{1} S_{1}+\cdots+p_{a_{n}} S_{a_{n}}+p_{a_{n}+1} S_{a_{n}+1}+\cdots+p_{n} S_{n}\right\} \\
= & \frac{1}{P_{n}}\left\{p_{1} S_{1}+\cdots+p_{n^{(1)}} S_{n^{(1)}}\right\}+\frac{P_{n^{(1)}+1}^{n}}{P_{n}} D_{n^{(1)}}^{n} R_{n} \quad \text { whenever } a_{n}=n^{(1)}, \\
R_{n}= & \frac{1}{P_{n}}\left\{p_{1} S_{1}+\cdots+p_{n^{(2)}} S_{n^{(2)}}+p_{n^{(2)}+1^{(2)}+1} S_{n^{(2)}}+\cdots+p_{n^{(1)}} S_{n^{(1)}}\right\}+\frac{P_{n^{(1)}+1}^{n}}{P_{n}} D_{n^{(1)}}^{n} R_{n} \\
= & \frac{1}{P_{n}}\left\{p_{1} S_{1}+\cdots+p_{n^{(2)}} S_{n^{(2)}}\right\}+\frac{P_{n^{(2)}+1}^{n^{(1)}}}{P_{n}} D_{n^{(2)}}^{n^{(1)}} R_{n^{(1)}} \\
& +\frac{P_{n^{(1)}+1}^{n}}{P_{n}} D_{n^{(1)}}^{n} R_{n} \quad \text { whenever } a_{n^{(1)}}=n^{(2)} .
\end{aligned}
$$

If we continue this until we come to some positive integer $N$ that depends on $n$, then we observe that

$$
\begin{aligned}
R_{n}= & \frac{P_{n^{(N+1)}+1}^{n^{(N)}}}{P_{n}} D_{n^{(N+1)}}^{n^{(N)}} R_{n^{(N)}}+\frac{P_{n^{(N)}+1}^{n^{(N-1)}}}{P_{n}} D_{n^{(N)}}^{n^{(N-1)}} R_{n^{(N-1)}}+\cdots \\
& +\frac{P_{n^{(2)}+1}^{n^{(1)}} D_{n^{(2)}}^{n^{(1)}} R_{n^{(1)}}+\frac{P_{n^{(1)}+1}^{n}}{P_{n}} D_{n^{(1)}}^{n} R_{n}}{P_{n}}
\end{aligned}
$$

for each $n$ whenever $n^{(N)} \geq 1$ and $n^{(N+1)}=0$. The above relation may be considered as a transformation of the form $\sigma_{n}$ which carries $\left(D_{a}^{n} R_{n}\right)$ into $\left(R_{n}\right)$. This transformation provides the conditions (19) and (20) by virtue of $n^{(r)}>n^{(r+1)}, r=1,2, \ldots, N$, and $n^{(N+1)}=0$. Furthermore, for a fixed $k$, the coefficient of $\left(D_{a_{k}}^{k} R_{k}\right)$ is either zero or a fractional expression of which the denominator is $P_{n}$ and the numerator is $\leq P_{k}$. Therefore (18) is satisfied. 
According to this, we get $\lim _{n \rightarrow \infty} R_{n}=u$ in view of $\lim _{n \rightarrow \infty} D_{a}^{b} R_{n}=u$ by considering the Silverman-Toeplitz theorem [13] and the proof is completed.

Let $d_{n, k}$ and $c_{n, k}$ be different from each other for at most a finite set of values of $n$. We know that since the two transformations $\sigma_{n}=\sum_{k=1}^{\infty} d_{n, k} x_{k}$ and $\sigma_{n}^{1}=\sum_{k=1}^{\infty} c_{n, k} x_{k}$ are equivalent, we write the following corollary as a result of Theorem 4.2.

Corollary $4.3\left(D_{a}^{b} R, p\right) \subset(R, p)$ whenever $b_{n}=n$ for almost all $n$.

Taking into account Theorem 4.1 and Theorem 4.2, we can write the next result.

Theorem 4.4 $\left(D_{a}^{n} R, p\right) \sim(R, p)$ if and only if $\left(D_{a}^{n} R, p\right)$ is proper where the symbol $\sim$ denotes equivalence between transformations.

In the case $p_{n}=1$ for all $n$ Theorem 4.1, Theorem 4.2, and Theorem 4.4 give us Theorem 4.1, Theorem 6.1, and Theorem 6.2 in [1], respectively.

Theorem $4.5\left(D_{a}^{b} R, p\right) \subset(R, p)$ whenever $\left(b_{n}\right)$ includes almost all positive integers.

Proof Assume that the sequence $\left(S_{n}\right)$ is $\left(D_{a}^{n} R, p\right)$-summable to the sum $l$, i.e.

$$
\lim _{n \rightarrow \infty} D_{a}^{b} R_{n}=l
$$

and we choose an integer $N$ so large that $\left(b_{n}\right)$ includes all integers greater than $N$. Accordingly, we put $i_{1}=i_{2}=\cdots=i_{N}=1$ such that $b_{i_{n}}=n$ for each $n>N$. By virtue of $\lim _{n \rightarrow \infty} i_{n}=+\infty$ and $\lim _{n \rightarrow \infty} D_{a}^{b} R_{n}=l$ it is easily seen that $\lim _{n \rightarrow \infty} D_{a}^{b} R_{i_{n}}=l$. Therefore we see that $\left(S_{n}\right)$ is $D_{a_{i_{n}}}^{b_{i_{n}}}$-summable to $l$. Hence, we obtain the required result by considering Corollary 4.3.

\section{Competing interests}

The authors declare that they have no competing interests.

\section{Authors? contributions}

All authors read and approved the final manuscript.

\section{Acknowledgements}

The authors would like to express their thanks to the reviewers and editors for their helpful suggestions and advice. The first author was supported by the Council of Higher Education of Turkey under a Postdoctoral grant.

Received: 21 March 2014 Accepted: 17 December 2014 Published online: 15 January 2015

\section{References}

1. Agnew, RP: On deferred Cesáro means. Ann. Math. (2) 33(3), 413-421 (1932)

2. Armitage, DH, Maddox, IJ: A new type of Cesáro mean. Analysis 9, 195-204 (1989)

3. Deǧer, U, Daǧadur, I, Küçükaslan, M: Approximation by trigonometric polynomials to functions in $L_{p}$-norm. Proc. Jangjeon Math. Soc. 15(2), 203-213 (2012)

4. Değer, U, Kaya, M: On the approximation by Cesáro submethod. Palest. J. Math. 4(1), 44-56 (2015)

5. Prösdorff, S: Zur Konvergenz der Fourier reihen Hölder Stetiger Funktionen. Math. Nachr. 69, 7-14 (1975)

6. Alexits, G: Convergence Problems of Orthogonal Series. Pergamon, New York (1961)

7. Chandra, P: On the generalized Fejer means in the metric of the Hölder space. Math. Nachr. 109, 39-45 (1982)

8. Mohapatra, RN, Chandra, P: Degree of approximation of functions in the Hölder metric. Acta Math. Hung. 41(1-2), 67-76 (1983)

9. Das, G, Ghosh, T, Ray, BK: Degree of approximation of functions by their Fourier series in the generalized Hölder metric. Proc. Indian Acad. Sci. Math. Sci. 106(2), 139-153 (1996)

10. Leindler, L: On the uniform convergence and boundedness of a certain class of sine series. Anal. Math. 27, 279-285 (2001) 
11. Zhou, SP, Zhou, P, Yu, DS: Ultimate generalization to monotonicity for uniform convergence of trigonometric series. Sci. China Math. 53(7), 1853-1862 (2010)

12. Bernstein, SN: Constructive Function Theory. Izdat. Akad. Nauk SSSR. Complete Works (1931)-(1953) (in Russian). 627 p. Vol II, Moscow (1954)

13. Boos, J: Classical and Modern Methods in Summability. Oxford University Press, Oxford (2000)

Submit your manuscript to a SpringerOpen ${ }^{\circ}$ journal and benefit from:

- Convenient online submission

- Rigorous peer review

- Immediate publication on acceptance

- Open access: articles freely available online

- High visibility within the field

Retaining the copyright to your article

Submit your next manuscript at $\gg$ springeropen.com 